\title{
Low-energy $\mathrm{V} t_{2 g}$ orbital excitations in $\mathrm{NdVO}_{3}$
}

\author{
J Laverock ${ }^{1}$, B Chen ${ }^{1}$, A R H Preston ${ }^{1}$, D Newby, Jr ${ }^{1}$, \\ L F J Piper ${ }^{1,2}$, L D Tung ${ }^{3} \dagger$, G Balakrishnan ${ }^{3}$, P-A Glans ${ }^{4}$, \\ J-H Guo ${ }^{4}$ and K E Smith ${ }^{1,5}$ \\ ${ }^{1}$ Department of Physics, Boston University, 590 Commonwealth Avenue, \\ Boston, MA 02215, USA \\ 2 Department of Physics, Applied Physics and Astronomy, Binghamton \\ University, Binghamton, NY 13902, USA \\ ${ }^{3}$ Department of Physics, University of Warwick, Coventry, CV4 7AL, United \\ Kingdom \\ 4 Advanced Light Source, Lawrence Berkeley National Laboratory, Berkeley, CA \\ 94720, USA \\ ${ }^{5}$ School of Chemical Sciences and The MacDiarmid Institute for Advanced \\ Materials and Nanotechnology, The University of Auckland, Private Bag 92019, \\ Auckland 1142, New Zealand \\ E-mail: laverock@bu.edu \\ PACS numbers: 78.70.Ck,75.25.Dk,73.20.Mf
}

\begin{abstract}
The electronic structure of $\mathrm{NdVO}_{3}$ and $\mathrm{YVO}_{3}$ has been investigated as a function of sample temperature using resonant inelastic soft $\mathrm{x}$-ray scattering at the $\mathrm{V} \mathrm{L}_{3}$-edge. Most of the observed spectral features are in good agreement with an atomic crystal-field multiplet model. However, a low energy feature is observed at $\sim 0.4 \mathrm{eV}$ that cannot be explained by crystal-field arguments. The resonant behaviour of this feature establishes it as due to excitations of the $\mathrm{V} t_{2 g}$ states. Moreover, this feature exhibits a strong sample temperature dependence, reaching maximum intensity in the orbitally-ordered phase of $\mathrm{NdVO}_{3}$, before becoming suppressed at low temperatures. This behaviour indicates that the origin of this feature is a collective orbital excitation, i.e. the bi-orbiton.
\end{abstract}

$\dagger$ Present address: Department of Physics \& Astronomy, University College London, Gower Street, London WC1E 6BT, UK 


\section{Introduction}

The competition and interplay between spin, orbital and charge degrees of freedom in transition metal oxides yield extremely rich, and often unexpected, phase diagrams[1]. In contrast to $e_{g}$ active systems, such as the manganites, partially filled $t_{2 g}$ electron systems experience weaker coupling to the lattice, leading to weaker Jahn-Teller distortions. Alongside the strong coupling between spin and orbital degrees of freedom in $t_{2 g}$ systems, this leads to a wealth of near-degenerate spin- orbital- and chargeordered structures that are relevant at similar temperatures. In particular, in the rare-earth orthovanadates, $R \mathrm{VO}_{3}(R=\mathrm{Y}, \mathrm{La}-\mathrm{Lu})$, both orbital order $(\mathrm{OO})$ and antiferromagnetic spin order (SO) develop in at least three distinct ordered phases, concomitant with structural transitions and Jahn-Teller distortions[2, 3].

Below the $\mathrm{OO}$ transition temperature $\left(T_{\mathrm{OO}} \sim 200 \mathrm{~K}\right)$, the $\mathrm{V} t_{2 g}$ electrons in $R \mathrm{VO}_{3}$ become orbitally ordered in a G-type (G-OO) arrangement[3], in which $d_{x y}$ orbitals are always occupied and the $d_{y z}$ and $d_{z x}$ orbitals are alternately occupied. Below the Néel temperature, $T_{N} \sim 120 \mathrm{~K}$, C-type SO (C-SO) sets in, retaining the G-OO, and in which the spins are aligned antiferromagnetically in the $a b$ plane, and ferromagnetically along $c$. For the smaller rare-earth ions (Y, Dy - Lu), an additional transition occurs below $\sim 80 \mathrm{~K}$, in which the $\mathrm{OO}$ switches to C-type (C-OO) and the $\mathrm{SO}$ to G-type (G-SO)[3]. More recently, there has been some evidence that C-OO coexists with G-OO below $T_{N}$ for some of the larger rare-earths, including $\mathrm{NdVO}_{3}$, leading to phase-separated regions that grow from small droplets $[4,5]$. Optical measurements have observed a substantial temperature dependence in opticallyallowed $d d^{*}$ excitations, at energies of $1.8,2.4$ and $3.3 \mathrm{eV}[6,7]$. Although on-site $d d^{*}$ transitions are optically forbidden, dimer-type excitations, $d^{2} d^{2} \rightarrow d^{3} d^{1}$, are allowed, and the optical excitations have been interpreted in terms of the $d^{3}$ multiplet structure[7]. Subsequent time domain spectroscopy measurements have suggested the low-energy excitation is a Hubbard exciton, i.e. a bound exciton across the MottHubbard gap[8]. At lower energies, optical conductivity measurements have found a peak at $\sim 0.4 \mathrm{eV}$, i.e. within the Mott-Hubbard gap, which the authors attribute to a collective orbital excitation[9] in the form of a bi-orbiton[10]. Although previous orbiton assignments of optical data in $\mathrm{RVO}_{3}$ have been controversial (see, for example, Refs. $[11,12]$ ), such excitations have previously been observed at similar energies in the $d^{1}$ titanates with both optical spectroscopy[13] and resonant inelastic x-ray scattering (RIXS)[14]. In the cuprates, the momentum dispersion of the orbiton has recently been revealed by high-resolution RIXS, providing unambiguous evidence of their origin[15].

RIXS is fast developing as a powerful and valuable probe of correlated electron systems (e.g. Refs. $[15,16]$ ). Transition metal $L$-edge RIXS is a bulk sensitive, direct two-step process[17], in which the incident photon is resonantly tuned to a feature of the absorption spectrum, i.e. a core level transition, leading to the unambiguous association of RIXS features with a particular atomic site and even a particular orbital. The specific direct RIXS process at the $\mathrm{V} L$-edge in $R \mathrm{VO}_{3}$ can be summarised as, $2 p^{6} 3 d^{2} \rightarrow 2 p^{5} 3 d^{3} \rightarrow 2 p^{6} 3 d^{2 *}$, where the ${ }^{*}$ in the final state indicates it may be an excited state or configuration of the ion. For example, $d d^{*}$ transitions include excitations of the $3 d$ electrons both within the $\mathrm{V} t_{2 g}$ manifold, and between $t_{2 g}$ and $e_{g}$ states. Moreover, unlike optical measurements, soft x-rays carry appreciable momentum, and can probe excitations away from the zone centre. Here, we present a detailed study of the RIXS excitations of the $\mathrm{V} t_{2 g}$ electrons of $\mathrm{NdVO}_{3}$, demonstrating that a low energy feature at $\sim 0.4 \mathrm{eV}$ may be associated with the delocalised orbital 
excitation of the $\mathrm{V} t_{2 g}$ electrons.

\section{Methods}

High-quality single crystals of $\mathrm{NdVO}_{3}$ and $\mathrm{YVO}_{3}$ were grown by the floating zone technique as described in Ref. [18]. Soft x-ray spectroscopy measurements were carried out at beamline X1B of the National Synchrotron Light Source, Brookhaven and the AXIS endstation of beamline 7.0.1 at the Advanced Light Source, Berkeley. Samples were cleaved ex-situ, and loaded into ultra-high vacuum within 10 minutes to ensure fresh, clean surfaces. X-ray absorption spectroscopy (XAS) measurements were performed in total electron yield (TEY) mode with an incident photon energy resolution $\left(\Delta E_{\text {in }}\right)$ of $0.2 \mathrm{eV}$ at FWHM, and the photon energy was calibrated using $\mathrm{TiO}_{2}$ reference spectra of the Ti $L$-edge and $\mathrm{O} K$-edge. RIXS spectra were recorded with a Nordgren-type spectrometer[19], and the instrument was calibrated using a $\mathrm{Zn}$ reference spectrum. The incident photon was polarised in the scattering plane $(\pi$ scattering geometry), and the scattering angle was $90^{\circ}$, with the surface normal at $45^{\circ}$ to the incident photons. All spectra used to construct the RIXS intensity map of $\mathrm{NdVO}_{3}$ were recorded during the same sample conditions with spectrometer resolution $\left(\Delta E_{\mathrm{spec}}\right)$ and $\Delta E_{\text {in }}$ of $0.36 \mathrm{eV}$ at FWHM[20]. Additional, separate, measurements on $\mathrm{NdVO}_{3}$ and $\mathrm{YVO}_{3}$ were performed at several temperatures between $80 \mathrm{~K}$ and room temperature at higher resolution, with $\Delta E_{\mathrm{spec}}=0.26 \mathrm{eV}$ and $\Delta E_{\text {in }}=0.21 \mathrm{eV}$ (with combined resolution $\approx 0.33 \mathrm{eV}$ ). In these measurements, the incident photon energy was kept fixed to ensure quantitative comparison between spectra. Since the natural pixel width of these measurements is just one-half of the FWHM of the resolution function, we have employed sub-pixel sampling to these higher resolution measurements (see Appendix for details). For presentation purposes, and since error bars are difficult to quantify in this process, we show error bars at the natural pixel width of the measurement, with the sub-pixel sampled data shown as a 'guide for the eye'.

\section{Results}

$\mathrm{V} L_{3,2}$-edge XAS spectra are shown in Fig. 1(a), and are in very good agreement with similar measurements of $\mathrm{YVO}_{3}[21,22]$, as well as with atomic crystal field multiplet (CFM) calculations of the $d^{2} \mathrm{~V}^{3+}$ ion, shown in tetragonal $D_{4 h}$ symmetry at the bottom of the figure. Since the $e_{g}$ states are well separated from the $t_{2 g}$ states in $R \mathrm{VO}_{3}$, we choose combinations of the crystal field $(\mathrm{CF})$ parameters $D_{s}$ and $D_{t}$ that preserve the degeneracy of the $e_{g}$ levels, leaving just a single parameter that characterises the lowering in symmetry between $O_{h}$ and $D_{4 h}$. This parameter, $\delta$ $\left(D_{s}=-\delta / 7\right.$ and $\left.D_{t}=4 \delta / 35\right)$, represents the splitting between the $d_{x y}$ and $d_{x z, y z}$ states, and is illustrated in the inset to Fig. 1(b). The CFM spectrum in Fig. 1(a) is calculated with $10 D q=2.0 \mathrm{eV}$ and $\delta=0.1 \mathrm{eV}$, approximately accounting for the lowering in energy of the $d_{x y}$ level by $0.1-0.2 \mathrm{eV}$ that is anticipated by first-principles studies $[24,23]$ and which has recently been observed directly in high-resolution RIXS measurements of $\mathrm{YVO}_{3}[22]$. From the CFM calculations, we associate the shoulder around $515 \mathrm{eV}$ predominantly with excitations within the $t_{2 g}$ states, and the peak at $516.5 \mathrm{eV}$ with excitations into the $e_{g}$ states. The experimental XAS spectra are found to be relatively insensitive to temperature; for example, the room temperature spectra are slightly broader, with a larger contribution $\sim 517.5 \mathrm{eV}$ and a weaker intensity of 

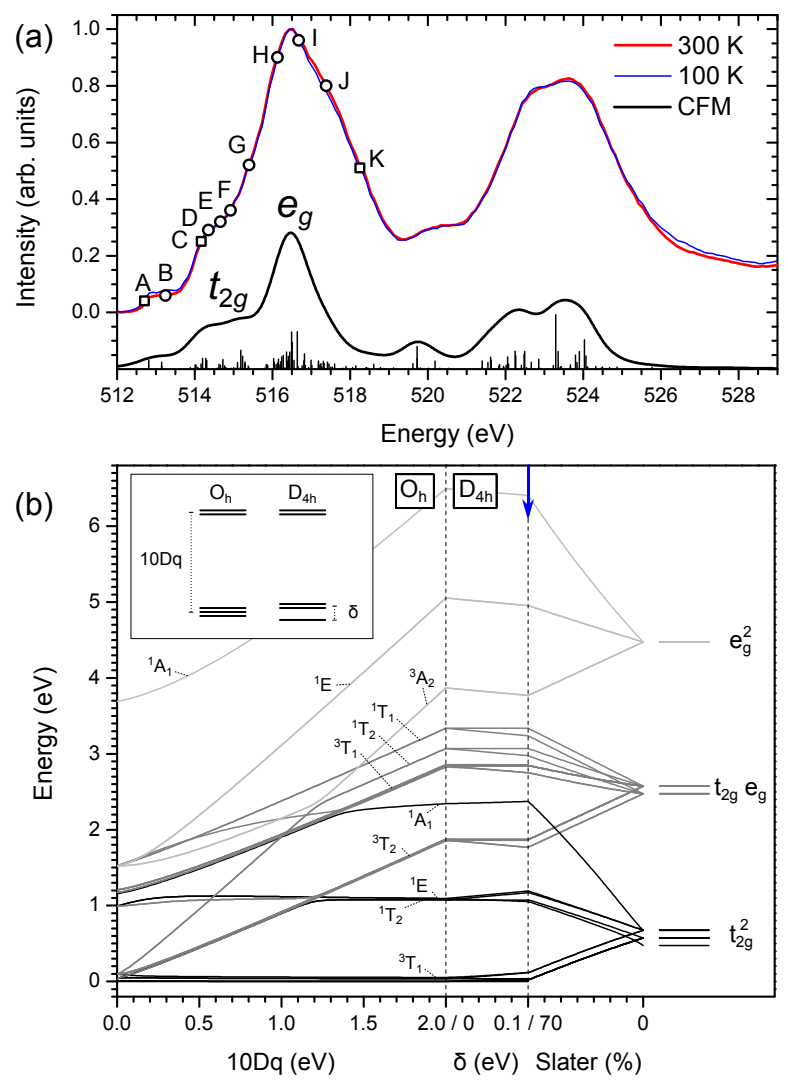

Figure 1. (a) $\mathrm{V} L_{3,2}$-edge XAS spectra of $\mathrm{NdVO}_{3}$ at room temperature and $100 \mathrm{~K}$, compared with the atomic CFM model $(10 D q=2.0 \mathrm{eV}, \delta=0.1 \mathrm{eV}$, and Slater reduction of $70 \%$ ). (b) Atomic multiplet energy level diagram for the $\mathrm{V}^{3+}$ $d^{2}$ ion in $O_{h}$ symmetry for $0 \leq 10 D q \leq 2.0 \mathrm{eV}$, followed by their splitting with $0 \leq \delta \leq 0.1 \mathrm{eV}$ in $D_{4 h}$ symmetry. The high-field parentage is shown on the right, corresponding to the reduction of the Slater integrals from $70 \% \rightarrow 0$. The inset schematically illustrates the CF energies, and definition of $\delta$.

the pre-edge features at $\sim 513 \mathrm{eV}$. We associate these very slight differences (which are reversible and reproducible) with the small change in the local $\mathrm{CF}$ between the high temperature orthorhombic phase and the low temperature monoclinic phase[23].

In order to separate localised CF $\left(d d^{*}\right)$ transitions from delocalised excitations in the RIXS data, calculations of the multiplet configurations have been performed in both octahedral $\left(O_{h}\right)$ and $D_{4 h}$ symmetry, shown in Fig. 1(b). In wide band-gap insulators (such as $R \mathrm{VO}_{3}$ ), local CF $\left(d d^{*}\right)$ transitions in RIXS are described well by the CFM model[25, 26]. On the left of Fig. 1(b), the evolution in multiplet energies are shown as $10 D q$ is increased from zero (the free ion picture) to $2.0 \mathrm{eV}$ in $O_{h}$, including spin-orbit coupling (which weakly splits some of the lines). Subsequently, the $\delta$ parameter is increased in $D_{4 h}$ symmetry to $0.1 \mathrm{eV}$, approximating the CF of $R \mathrm{VO}_{3}$ [and indicated by the arrow in Fig. 1(b)]. Finally, the Slater integrals are reduced to zero, reflecting the single-particle parentage of the states (i.e. $t_{2 g}^{2}, t_{2 g}^{1} e_{g}^{1}$ and $e_{g}^{2}$ ). In this picture, the $t_{2 g}^{2}$ occupation is further split into three levels: $d_{x y}^{2}$, $d_{x y}^{1} d_{x z, y z}^{1}$ and $d_{x z, y z}^{2}$. 


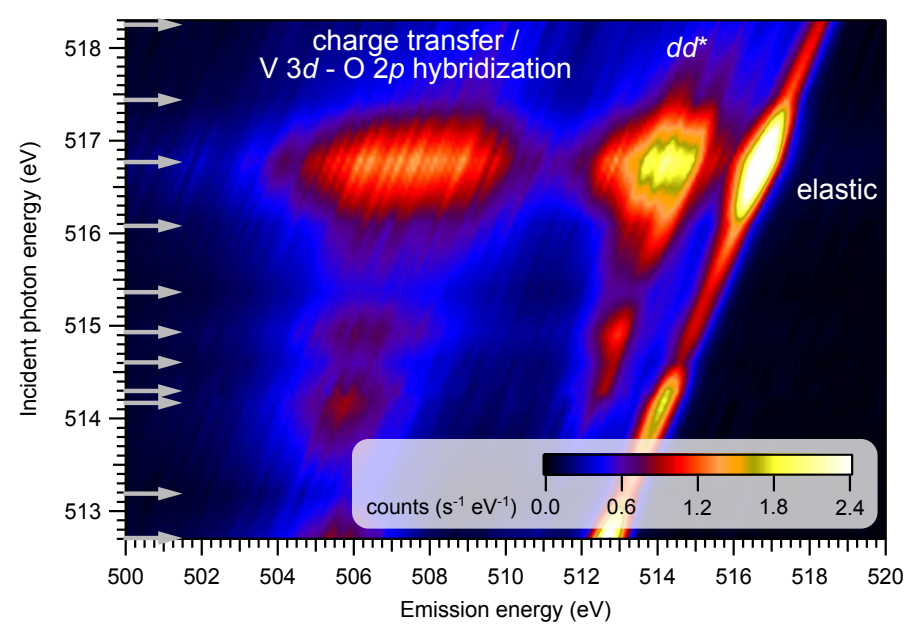

Figure 2. RIXS intensity map of $\mathrm{NdVO}_{3}$ at room temperature. The discrete incident photon energies used to construct the intensity maps are shown by the arrows at the left of the figure. The colour intensity scale represents the experimental transition rate.

The $\mathrm{V} L_{3}$-edge RIXS measurements are summarised in Fig. 2, shown as an intensity map constructed from eleven discrete incident photon energies between 512.5 and $518.5 \mathrm{eV}$ [indicated in Fig. 1(a)]. Below resonance, the spectra consist of quasi-elastic scattering (including very low energy phonon contributions), with a weak contribution from charge-transfer processes centered $\approx 7 \mathrm{eV}$ lower in energy, in good agreement with estimations based on our photoemission measurements (not shown). As the incident photon energy is increased, the inelastic RIXS processes (i.e. $d d^{*}$ excitations) become more intense, developing into local maxima at $\sim 514.5 \mathrm{eV}$ and $\sim 516.5 \mathrm{eV}$, corresponding to excitations into unoccupied $t_{2 g}$ and $e_{g}$ states, respectively [as illustrated in Fig. 1(a)]. Therefore, we can associate $d d^{*}$ loss features at the first of these resonances (near $514.5 \mathrm{eV}$ ) predominantly with excitations within the $t_{2 g}$ manifold, whereas features at the second resonance (near $516.5 \mathrm{eV}$ ) are associated to a greater extent with excitations into the $e_{g}$ states.

In Fig. 3, five representative spectra are shown on an energy transfer scale at room temperature and at $100 \mathrm{~K}$, and are broadly in good agreement with the recent high-resolution RIXS data of Ref. [22] on $\mathrm{YVO}_{3}$. The excitation energies used for the two different temperatures have been carefully checked to agree to within $\pm 0.1 \mathrm{eV}$. Spectra (D-F), separated by just $0.6 \mathrm{eV}$, correspond to energies at the $t_{2 g}$ absorption resonance, whereas spectra $(\mathrm{H})$ and $(\mathrm{J})$ are recorded near the $e_{g}$ resonance. Note that there is only a very weak dependence of the intensity of the $d d^{*}$ transitions with temperature between the disordered phase $(300 \mathrm{~K})$ and spin- and orbitally-ordered (GOO, C-SO) phase at $100 \mathrm{~K}$. Through comparison with the CFM model of Fig. 1(b), features I and III can be associated with singlet $t_{2 g}^{2}$ excitations, and II is a triplet transition into a $t_{2 g}^{1} e_{g}^{1}$ excited state. At higher energies, IV represents a broad group of $t_{2 g}^{1} e_{g}^{1}$ transitions, and V and VI are $e_{g}^{2}$ final states. The symmetries of these excitations (in $O_{h}$ and neglecting spin-orbit coupling) are labelled in Fig. 3. We emphasise that the ${ }^{1} T_{2} /{ }^{1} E$ transition in Fig. $1(\mathrm{~b})$ is relatively insensitive to the $\mathrm{CF}$, and is primarily determined by $2 J_{H}=2(3 B+C)$ [27], where $J_{H}$ is the on-site Hund's exchange parameter, and $B$ and $C$ are the Racah parameters. This transition can 


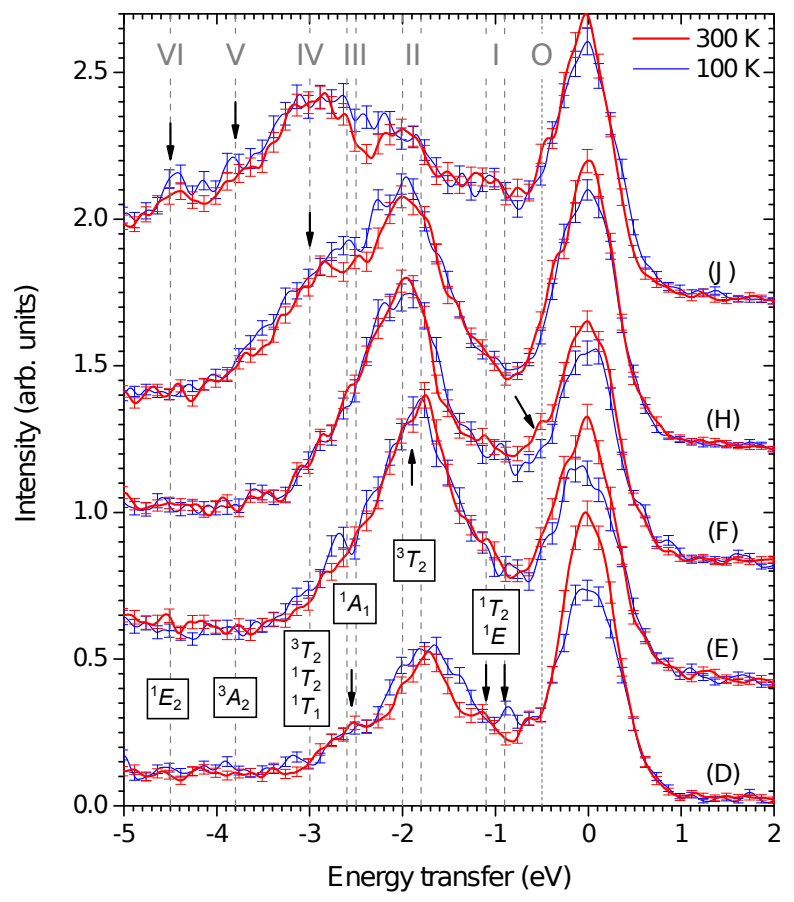

Figure 3. Representative RIXS spectra at room temperature and $100 \mathrm{~K}$.

therefore be used as an independent measurement of $J_{H}$ and $B$, irrespective of the agreement of the CFM model elsewhere, yielding $J_{H}=0.55 \mathrm{eV}$ and $B=0.083 \mathrm{eV}$, which are similar to, but smaller than, typical values used in theoretical models of the orbital order[28, 23].

Having established the origin of the CF excitations in the RIXS spectra, we now turn to the feature that cannot be explained by the CFM model. At $-0.4 \mathrm{eV}$, a weak feature ' $\mathrm{O}$ ' is present for several different incident energies at room temperature. This feature is found to resonate (have largest intensity) at the $t_{2 g}$ absorption resonance, indicating its origin is excitations of the $\mathrm{V} t_{2 g}$ states, and is most apparent in spectrum (F). At $100 \mathrm{~K}$, it becomes indistinguishable from elastic scattering. This feature is not expected from the CFM model, and we now investigate its origin through higher resolution $(\approx 0.33 \mathrm{eV}$ combined resolution), temperature-dependent measurements. In Fig. 4(a), five spectra between room temperature and $80 \mathrm{~K}$ are shown at a single incident photon energy of $514.5 \mathrm{eV}$. This incident photon energy [close to spectrum (E) in Fig. 3] is chosen to maximise the contribution from ' $\mathrm{O}$ ' (resonant with $t_{2 g}$ absorption). The quasi-elastic scattering is strong, even at this energy, and partially obscures ' $\mathrm{O}$ ' in its tails, although some asymmetry of this feature is clear, particularly at $160 \mathrm{~K}$. In order to clarify the temperature behaviour of ' $\mathrm{O}$ ', the quasi-elastic peak has been subtracted from the spectra in Fig. 4(a) [an example of this subtraction is shown in the lower inset to Fig. 4(a)]. Owing to contribution from other low-energy loss processes, such as phonons and low-energy CF excitations[22], the elastic signal is fitted to a Voigt lineshape only above $0 \mathrm{eV}$, corresponding only to instrumentbroadened elastic scattering. In Fig. 4(a), the linewdith of the quasi-elastic peak has been fitted to each spectrum independently, accounting for the temperature-dependent evolution in the phonon contribution and thermal population of the CF ground state. 

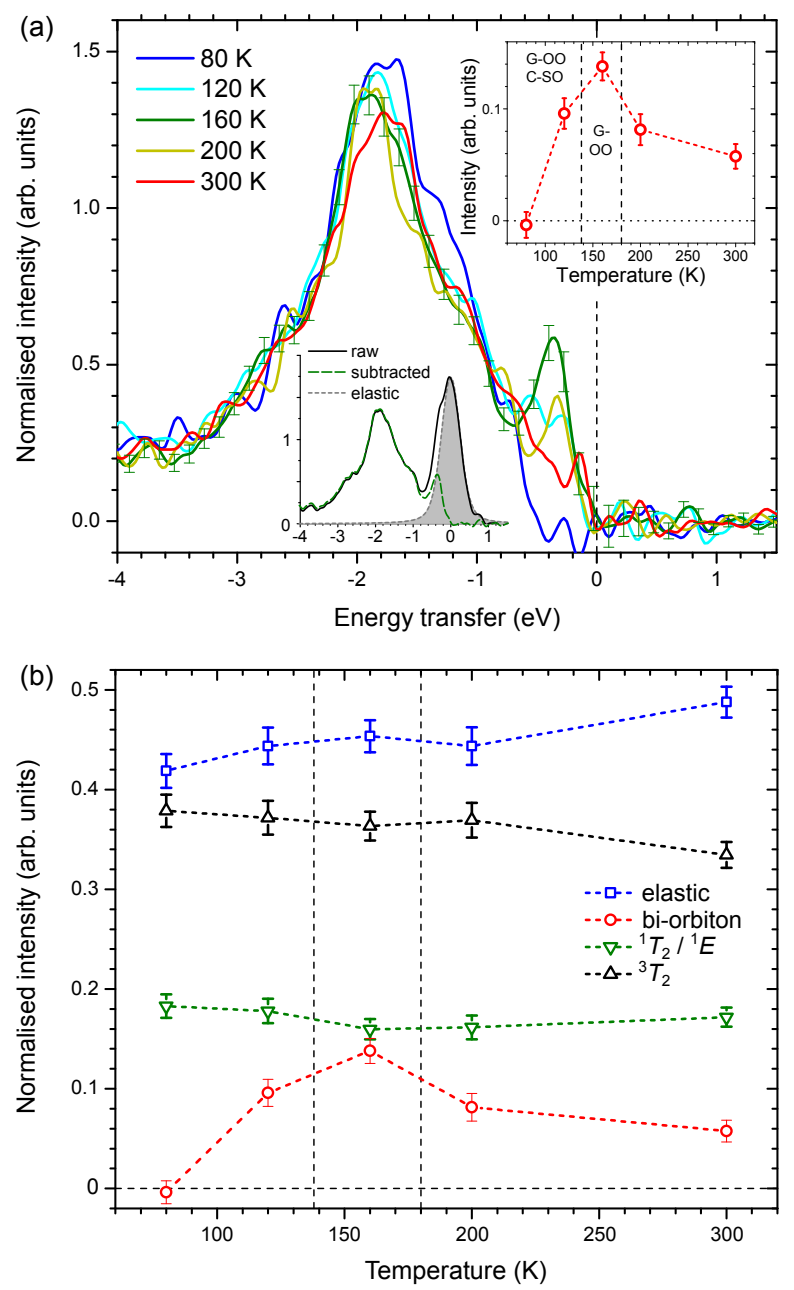

Figure 4. Temperature dependence of RIXS excitations in $\mathrm{NdVO}_{3}$, recorded with an incident photon energy of $514.5 \mathrm{eV}$. (a) Spectra after removal of the elastic peak, showing the peak at $-0.4 \mathrm{eV}$ that cannot be explained by the CFM model. Representative error bars are shown for one of the spectra. An example of the elastic peak subtraction is shown in the lower inset. The upper inset shows the integrated intensity of the spectra between -0.55 and $-0.25 \mathrm{eV}$. (b) Temperature dependence of the RIXS excitations in (a). The intensity is calculated in a $0.3 \mathrm{eV}$ window centered about $0 \mathrm{eV}$ (elastic peak), $-0.4 \mathrm{eV}$ (bi-orbiton, after elastic peak subtraction), $-1.0 \mathrm{eV}\left({ }^{1} T_{2} /{ }^{1} E\right)$ and $-1.9 \mathrm{eV}\left({ }^{3} T_{2}\right)$. The horizontal dotted line corresponds to zero intensity, and the vertical dashed lines indicate $T_{\mathrm{OO}}$ and $T_{N}[3]$.

However, we emphasise that the following conclusions are insensitive to such details, and we obtain similar results if a single (mean) linewidth is used. The subtracted spectra contain a large temperature-dependent component centered at -0.4 , which peaks at $160 \mathrm{~K}$, before diminishing to zero at $80 \mathrm{~K}$. The inset to Fig. 4(a) displays the integrated intensity between -0.55 and $-0.25 \mathrm{eV}$ of each spectrum, illustrating the large relative contribution of this feature in the OO phase, before its suppression in the $\mathrm{SO} / \mathrm{OO}$ phase. Even at room temperature, this component has a reasonably 
large contribution. Although low-energy local CF excitations are expected[23] below $-0.2 \mathrm{eV}$, they are observed to be relatively weak, compared with elastic scattering, in $\mathrm{YVO}_{3}$, and do not display appreciable temperature dependence[22]. Moreover, most of the spectral weight that contributes to the temperature dependence observed in Fig. 4(a) is concentrated well above $-0.2 \mathrm{eV}$. In Fig. 4(b), this temperature dependence is compared with the temperature dependence of elastic scattering and local $\mathrm{CF}$ excitations. As anticipated by Fig. 3, the elastic scattering and local CF transitions above $80 \mathrm{~K}$ are relatively insensitive to the orbital and spin order, showing only weak (and approximately linear) variations in intensity with temperature, in contrast to the pronounced temperature dependence of the $-0.4 \mathrm{eV}$ feature. As we discuss in more detail below, the spectrum recorded at $80 \mathrm{~K}$ exhibits quite different $\mathrm{CF}$ transition probabilities to the other spectra, with more weight appearing at -1.7 and $-1.2 \mathrm{eV}$.

Before we discuss the origin of the low-energy excitation in more detail, we briefly reinforce its presence through non-parameterised analysis. In Fig. 5(a), the RIXS map of Fig. 2 has been integrated over the $t_{2 g}$ and $e_{g}$ resonances, yielding a (weighted) summed spectrum on an energy transfer scale. Here, fluorescent features (which are dispersive in energy transfer) are spread out and contribute a broad 'background', whereas RIXS features reinforce. A slight asymmetry of the elastic peak in Fig. 5(a) develops into a prominent shoulder at $-0.4 \mathrm{eV}$ after treatment with a maximumentropy deconvolution technique (MaxEnt)[20]. At the bottom of Fig. 5(a), the gradient of this MaxEnt spectrum is shown, in which the shoulder centered at $-0.4 \mathrm{eV}$ corresponds to feature ' $\mathrm{O}$ '.

We now focus on the temperature-dependent data of Fig. 4, and employ difference spectra to examine the low-energy feature. Since the quasi-elastic peak intensity evolves with temperature, we choose two temperatures that are close together (120 and $160 \mathrm{~K}$ ) to reduce problems associated with the variation of the CF population with temperature. Owing to the increased noise level of difference spectra, the raw spectra of Fig. 4 are first passed through a noise filter[26] before subtraction, and are shown in Fig. 5(b). The difference spectrum is shown at the bottom of Fig. 5(b), illustrating the excess intensity in the $160 \mathrm{~K}$ spectrum at $-0.4 \mathrm{eV}$. For comparison, the difference in the raw spectra is also shown, exhibiting the same structure. In Fig. 5(c), the same two spectra have been treated with the MaxEnt deconvolution procedure, and the extra spectral weight at $-0.4 \mathrm{eV}$ can be directly seen. Finally, we note that the data presented in Fig. 5(a) and Figs. 5(b-c) were recorded several months apart on different cleaves of the sample boule, illustrating the reproducibility of the data.

\section{Discussion}

The above results indicate that the $-0.4 \mathrm{eV}$ feature is associated with the orbital order, but is strongly suppressed at low temperature. CF (multiplet) excitations can be ruled out: low-energy multiplet excitations occur on the order of the parameter $\delta$ [illustrated in the inset to Fig. 1(b)], and are not expected as large as $0.4 \mathrm{eV}$. In $R \mathrm{TiO}_{3}$, whose CF splitting is roughly twice as large as the vanadates[23, 29], local $\mathrm{CF}$ excitations were predicted below $300 \mathrm{meV}$ [14]. Moreover, it is hard to reconcile the temperature evolution shown in Fig. 4(b) with local CF excitations. Secondly, the lowest energy dimer excitations are expected at $3 J_{H}[30]$, and are observed in optical spectra at $1.8 \mathrm{eV}[6]$, and can therefore also be ruled out. Finally, the energy is well above that of phonon and magnon modes, which occur below $100 \mathrm{meV}$ in $R \mathrm{VO}_{3}$ [11].

Orbital excitations (in the form of bi-orbitons) have previously been observed with 

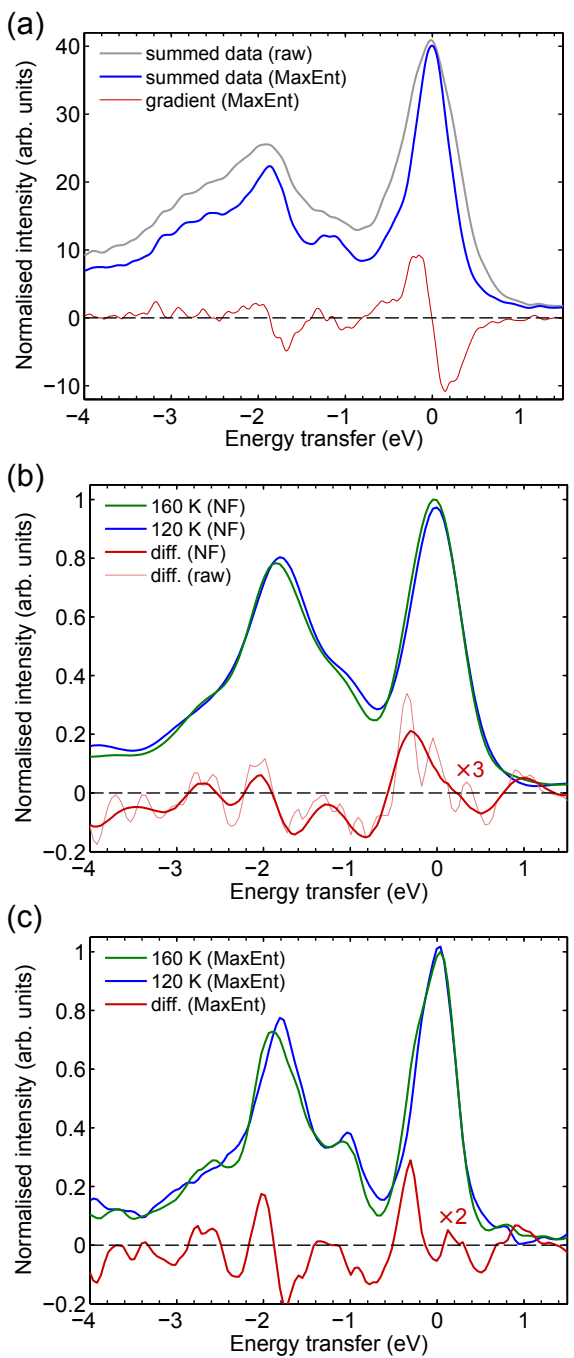

Figure 5. Parameter-free analysis of $\mathrm{V} L_{3}$-edge RIXS spectra of $\mathrm{NdVO}_{3}$ showing the presence of a weak feature at $\approx-0.4 \mathrm{eV}$. (a) Summed spectra at room temperature, obtained by integrating the RIXS map of Fig. 2 between 514.7 and $517.3 \mathrm{eV}$, i.e. over the $t_{2 g}$ and $e_{g}$ resonances. The summed spectrum has also been deconvoluted using the MaxEnt procedure, and the gradient of this spectrum is also shown. (b) Difference between the $160 \mathrm{~K}$ and $120 \mathrm{~K}$ spectra of Fig. 4 after applying a noise filter (NF) to the data. For comparison, the difference between the raw spectra is also shown. (c) The same $160 \mathrm{~K}$ and $120 \mathrm{~K}$ spectra after being treated by the MaxEnt deconvolution procedure. Their difference is also shown for comparison.

RIXS at the Ti $L_{3}$-edge of $\mathrm{LaTiO}_{3}$ and $\mathrm{YTiO}_{3}$ at an energy of $0.25 \mathrm{eV}$ [14], following an earlier observation of a similar feature in optical measurements[13]. These excitations were described well by a superexchange model, in which the bi-orbiton process (which displays only very weak dispersion) was found to be of stronger intensity in the RIXS spectrum than single orbiton modes. In $\mathrm{YVO}_{3}$ and $\mathrm{HoVO}_{3}$, optical measurements exhibit a strong peak at $0.4 \mathrm{eV}$, which the authors attribute to the collective bi- 


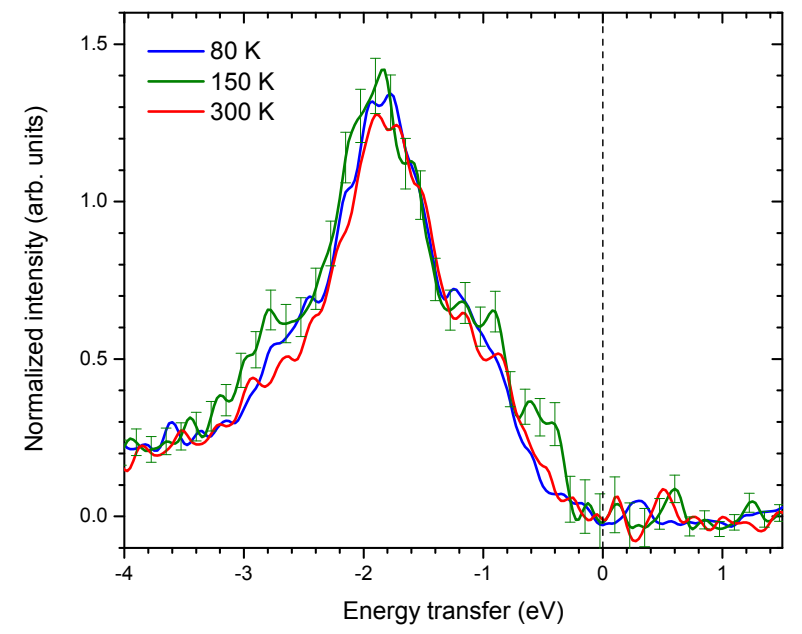

Figure 6. Temperature dependence of RIXS excitations in $\mathrm{YVO}_{3}$, recorded with an incident photon energy of $514.5 \mathrm{eV}$. The spectra are shown after removal of the elastic peak, in the same way as Fig. 4(a).

orbiton[10]. This feature, present at room temperature, has largest intensity in the G-OO phase before being suppressed in the C-OO phase of $\mathrm{YVO}_{3}$ due to the specific low-temperature orbital ordering pattern. In their model of the bi-orbiton, $d_{x z, 1}$ and $d_{y z, 2}$ orbitals on neighbouring sites 1 and 2 are excited to $d_{y z, 1}$ and $d_{x z, 2}$, conserving the spin order in the process[10]. This is allowed in the G-OO/C-SO pattern, but forbidden in the $\mathrm{C}-\mathrm{OO} / \mathrm{G}-\mathrm{SO}$ pattern, explaining the temperature dependence of the optical spectra. Our measurements reveal a similar behaviour with temperature, in which feature ' $\mathrm{O}$ ' grows in intensity in the intermediate G-OO phase, and persists into the G-OO/C-SO phase transition $\left(T_{N}=138 \mathrm{~K}\right)$. Below $T_{N}$, however, recent high-resolution diffraction measurements have suggested a coexistence of G-OO and $\mathrm{C}-\mathrm{OO}$ for rare earth ions of intermediate size (including $\left.\mathrm{NdVO}_{3}\right)[4,5]$. The fraction of $\mathrm{C}-\mathrm{OO}$ in this phase-separated region is suggested to be as high $65 \%$ for $\mathrm{GdVO}_{3}$ and $\mathrm{TbVO}_{3}[5]$. In $\mathrm{La}_{1-x} \mathrm{Lu}_{x} \mathrm{VO}_{3}$, the suppression of G-OO in favour of C-OO (due to cation disorder) has been found to result in large differences in the RIXS intensities of the ${ }^{3} T_{2}$ and ${ }^{1} T_{2} /{ }^{1} E$ excitations[31]. In Fig. 4(a), the transition probabilities of these features are observed to be notably different at $80 \mathrm{~K}$ compared with other temperatures, consistent with a growing fraction of C-OO coexisting with G-OO below $T_{N}$ in $\mathrm{NdVO}_{3}$. In the model of Ref. [10] this kind (C-type) of ordering would suppress the two-orbiton process, since it consists of nearest neighbours with the same spin but also the same orbital occupation. Even if the ratio of $\mathrm{C}-\mathrm{OO}$ is low, the formation of small droplets of C-OO within the G-OO matrix, as suggested by Ref. [4], would scatter the propagation of the delocalised orbital wave.

A very recent high-resolution RIXS study of $\mathrm{YVO}_{3}$ has also found a feature at $0.4 \mathrm{eV}$ in difference (between photon energies) spectra at the $\mathrm{O} K$-edge, which the authors link to the bi-orbiton[22]. However, although their $\mathrm{V} L_{3}$-edge RIXS spectra are in agreement with our $\mathrm{CF}$ energies, the $0.4 \mathrm{eV}$ feature was not observed at the $\mathrm{V}$ 
$L$-edge in their data, eliminating the possibility of directly associating this transition with $\mathrm{V}$ electrons. This also raises the question of why the bi-orbiton feature is observed in our $\mathrm{NdVO}_{3}$ data at the $\mathrm{V} L$-edge, but not by Ref. [22] in $\mathrm{YVO}_{3}$ at the same edge. In Fig. 6, we show similar temperature-dependent $\mathrm{V} L$-edge RIXS measurements at $514.5 \mathrm{eV}$ on $\mathrm{YVO}_{3}$, recorded with the same instrument settings and treated in the same way as the $\mathrm{NdVO}_{3}$ data of Fig. 4. Although some intensity is observed near $-0.4 \mathrm{eV}$, it is much weaker than in $\mathrm{NdVO}_{3}$, and its temperature dependence is only barely discernible. As pointed out by Pavarini and co-workers in the rare-earth titanates, the precise details of the electronic structure, and hence CF levels and orbital order patterns, of perovskite-like materials is very sensitive to the $A$-site hybridisation[29]. In particular, the hybridisation between the unoccupied $\mathrm{Y} 4 d$ and $\mathrm{V} t_{2 g}$ orbitals is likely quite different to that between the broader and energetically higher lanthanide $5 d$ bands and $\mathrm{V} t_{2 g}$. Our measurements suggest that although $\mathrm{YVO}_{3}$ is one of the most studied $R \mathrm{VO}_{3}$ compounds, it may not be representative of a typical rare earth ion, and that future high-resolution studies of orbital wave physics in these compounds may be more fruitful in other compounds.

\section{Conclusions}

To summarise, we have presented a comprehensive RIXS study of the excitation spectrum of $\mathrm{NdVO}_{3}$ above and below the $\mathrm{OO}$ and $\mathrm{SO}$ transitions. Whereas most of the experimental features show very weak temperature dependence and can be explained very well from an atomic CFM perspective, a low-energy feature is observed with an energy of $0.4 \mathrm{eV}$. The resonant behaviour of this peak confirms its origin as excitations of the $\mathrm{V} t_{2 g}$ electrons. This feature cannot be explained as a local CF excitation, and exhibits a strong temperature dependence consistent with the orbital wave model of Ref. [10]. In this model, this peak is due to a collective bi-orbiton excitation, which is allowed in the G-OO and G-OO/C-SO phases. Below $T^{*} \sim 125 \mathrm{~K}$, a finite fraction of $\mathrm{C}-\mathrm{OO}$ coexists with the G-OO, and suppresses the excitation and propagation of the bi-orbiton. Our measurements are consistent with this model, suggesting the $-0.4 \mathrm{eV}$ feature observed in $\mathrm{NdVO}_{3}$ may be associated with the bi-orbiton excitation. This feature is found to be heavily suppressed in similar RIXS measurements of $\mathrm{YVO}_{3}$. Future high-resolution and momentum-resolved RIXS experiments are encouraged to unambiguously rule on its origin, and should be focussed near the $t_{2 g}$ resonance of compounds other than $\mathrm{YVO}_{3}$.

\section{Acknowledgements}

The Boston University program is supported in part by the Department of Energy under Grant No. DE-FG02-98ER45680. The ALS, Berkeley, is supported by the U.S. Department of Energy under Contract No. DE-AC02-05CH11231. The NSLS, Brookhaven, is supported by the U.S. Department of Energy under Contract No. DEAC02-98CH10886. G.B. gratefully acknowledges financial support from EPSRC Grant $\mathrm{EP} / \mathrm{I} 007210 / 1$.

\section{Appendix: Sub-Pixel Sampling}

The emission spectrometer used in these measurements is a Nordgren-type Rowland circle spectrometer [19]. The x-rays are collected from the sample through an 
adjustable entrance slit assembly, after which they are diffracted by a spherical grating. The diffracted $\mathrm{x}$-rays are subsequently detected by a $2 \mathrm{D}$ multi-channel plate (MCP) detector. The image of the straight entrance slit through the spherical grating is curved, necessitating the 2D detection system (see, for example, Fig. 2 of Ref. [19]). However, this curvature also affords the possibility of sampling below the natural pixel width of the MCP detector.

Shown in Fig. 1(a) is the 2D image of the region near the elastic peak of a representative RIXS measurement, displaying the curved image of the elastic scattering, as well as a low-energy CF transition. The 2D image is binned into 1024 horizontal "energy" channels (actually evenly-spaced in wavelength), and 32 vertical "slices". Next to this image, in Fig. 1(b), iso-energy curves are shown for the same measurement at the same part of the detector. In addition to the obvious offset in energy between each slice, the individual slices each have their own (different) calibration (pixel width). It is clear that, in general, there is a non-integer offset (in pixels) between each slice, which means that the centres of each pixel do no align. When correcting for the curvature, one is therefore faced with two possibilities: 1) to share the counts of a pixel that is offset by a fractional amount with the bins either side (i.e. bilinear interpolation), which is the conventional approach, or 2) to sample the data below the natural pixel width, and take advantage of this extra fidelity.

Sub-pixel sampling does not improve the instrument resolution, of course, since this is limited by the optics of the detector and the width of the entrance slit. However, it does allow for higher data fidelity, which is useful in assessing whether low-energy features of the data are due to random noise, or intrinsic to the system under investigation. At broad resolution functions, there is little to be gained by sub-pixel sampling, since one is already sampling at a small fraction of the resolution function using the natural pixel width. However, in our measurements, we set the instrument resolution function to $0.26 \mathrm{eV} \mathrm{FWHM} \mathrm{(the} \mathrm{smallest} \mathrm{achievable} \mathrm{slit} \mathrm{width}$ with manageable count rates), and the natural pixel width of $0.13 \mathrm{eV}$ only samples the data at half of the resolution FWHM (or more than 1 sigma). By using sub-pixel sampling, we are able to increase the fidelity to one tenth of the FWHM (or one quarter of sigma). In Fig. 1(c), the centre in relative energy of each pixel is shown by the points, with the conventional natural pixel width indicated by the vertical solid lines, indicating the energy coverage of the MCP detector. The vertical dashed lines correspond to sub-pixel sampling every fifth of a pixel, with many pixels contributing to each sampling point.

Owing to the varying pixel coverage of sub-pixel sampling, and the unknown errors introduced by the (bilinear) interpolation, a reliable error map is difficult to quantify at the sub-pixel level. Instead, in Figs. 4 and 5 of the manuscript, we have chosen to present the data using statistical error bars for the data points that are sampled at the natural pixel width, with the sub-pixel sampled data points shown as lines connecting these points (in effect, 'guides for the eye'). We believe this is the fairest way of the presenting this kind of analysis, where we make no direct claim on the error distribution of the sub-pixel sampled data points, but this information is provided as a qualitative impression of the higher fidelity that is available. Finally, the analysis of simulated data indicates that this approach does not introduce artefacts in the data. We emphasise that no cubic interpolants, or other high-order interpolation methods are used - only bilinear interpolation is used to distribute the counts (which is incapable, by itself, of developing peaks between data points). 

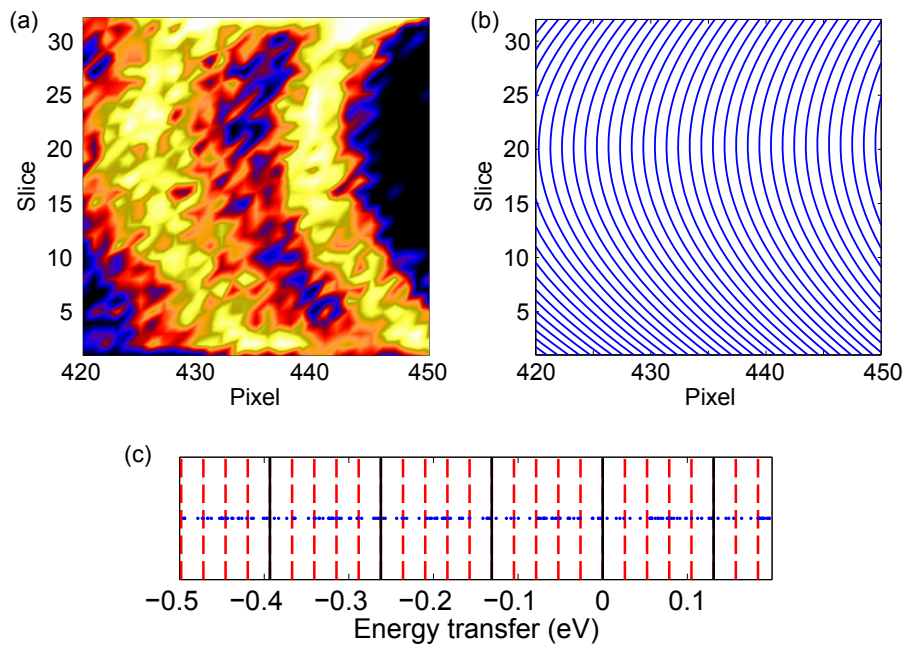

Figure 1. (a) Close-up of the image of the MCP of a typical $\mathrm{V} L_{3}$-edge RIXS measurement, illustrating the pronounced curvature in the detected image. (b) Iso-energy contours of the image shown in (a), showing the curvature function. (c) Pixel coverage of the MCP shown in (a) and (b). Each dot represents the centre in energy of one of the pixels in one of the slices in (a) and (b). The solid vertical lines represent the natural pixel width of the MCP, and the vertical dashed lines correspond to sub-pixel sampling at one-fifth of a pixel.

\section{References}

[1] Tokura Y and Nagaosa N 2000 Science 288 462; Mizokawa T, Khomskii D I and Sawatzky G A 1999 Phys. Rev. B 60 7309; van den Brink J, Khaliullin G and Khomskii D 1999 Phys. Rev. Lett. 835118.

[2] Blake G R, Palstra T T M, Ren Y, Nugroho A A and Menovsky A A 2001 Phys. Rev. Lett. 87 245501; Ulrich C, Khaliullin G, Sirker J, Reehuis M, Ohl M, Miyasaka S, Tokura Y and Keimer B 2003 Phys. Rev. Lett. 91257202.

[3] Miyasaka S, Okimoto Y, Iwama M and Tokura Y 2003 Phys. Rev. B 68 100406(R).

[4] Sage M H, Blake G R, Nieuwenhuys G J and Palstra T T M 2006 Phys. Rev. Lett. 96036401.

[5] Sage M H, Blake G R, Marquina C and Palstra T T M 2007 Phys. Rev. B 76195102.

[6] Tsvetkov A A, Mena F P, van Loosdrecht P H M, van der Marel D, Ren Y, Nugroho A A, Menovsky A A, Elfimov I S and Sawatzky G A 2004 Phys. Rev. B 69075110.

[7] Reul J, Nugroho A A, Palstra T T M and Grüninger M 2012 Phys. Rev. B 86125128.

[8] Novelli F, Fausti D, Reul J, Cilento F, van Loosdrecht P H M, Nugroho A A, Palstra T T M, Grüninger M and Parmigiani F 2012 Phys. Rev. B 86165135.

[9] Saitoh E, Okamoto S, Takahashi K T, Tobe K, Yamamoto K, Kimura T, Ishihara S, Maekawa S and Tokura Y 2001 Nature 410180.

[10] Benckiser E, Rückamp R, Möller T, Taetz T, Möller A, Nugroho A A, Palstra T T M, Uhrig G S and Grüninger M 2008 New J. Phys. 10053027.

[11] Miyasaka S, Onoda S, Okimoto Y, Fujioka J, Iwama M, Nagaosa N and Tokura Y 2005 Phys. Rev. Lett. 94 076405; Miyasaka S, Fujioka J, Iwama M, Okimoto Y and Tokura Y 2006 Phys. Rev. B 73224436.

[12] Sugai S and Hirota K 2006 Phys. Rev. B 73 020409(R).

[13] Ulrich C, Gössling A, Grüninger M, Guennou M, Roth H, Cwik M, Lorenz T, Khaliullin G and Keimer B 2006 Phys. Rev. Lett. 97157401.

[14] Ulrich C, Ament L J P, Ghiringhelli G, Braicovich L, Moretti Sala M, Pezzotta N, Schmitt T, Khaliullin G, van den Brink J, Roth H, Lorenz T and Keimer B 2009 Phys. Rev. Lett. 103 107205.

[15] Schlappa J, Wohlfeld K, Zhou K J, Mourigal M, Haverkort M W, Strocov V N, Hozoi L, Monney C, Nishimoto S, Singh S, Revcolevschi A, Caux J-S, Patthey L, Rønnow H M, van den Brink J and Schmitt T 2012 Nature 48582. 
[16] Braicovich L, Ament L J P, Bisogni V, Forte F, Aruta C, Balestrino G, Brookes N B, De Luca G M, Medaglia P G, Miletto Granozio F, Radovic M, Salluzzo M, van den Brink J and Ghiringhelli G 2009 Phys. Rev. Lett. 102 167401; Laverock J, Chen B, Smith K E, Singh R P, Balakrishnan G, Gu M, Lu J W, Wolf S A, Qiao R M, Yang W and Adell J 2013 Phys. Rev. Lett. 111047402.

[17] Ament L J P, van Veenendaal M, Devereaux T P, Hill J P and van den Brink J 2011 Rev. Mod. Phys. 83705.

[18] Tung L D, Lees M R, Balakrishnan G and Paul D McK 2007 Phys. Rev. B 75 104404; Tung L D, Lees M R, Balakrishnan G and Paul D McK 2007 Phys. Rev. B 76 064424; Tung L D, Ivanov A, Schefer J, Lees M R, Balakrishnan G and Paul D McK 2008 Phys. Rev. B 78 054416.

[19] Nordgren J and Nyholm R 1986 Nucl. Instrum. Meth. A 246242.

[20] Laverock J, Preston A R H, Newby Jr. D, Smith K E and Dugdale S B 2011 Phys. Rev. B bf 84235111.

[21] Pen H F, Abbate M, Fujimori A, Tokura Y, Eisaki H, Uchida S and Sawatzky G A 1999 Phys. Rev. B 597422.

[22] Benckiser E, Fels L, Ghiringhelli G, Moretti Sala M, Schmitt T, Schlappa J, Strocov V N, Mufti N, Blake G R, Nugroho A A, Palstra T T M, Haverkort M W, Wohlfeld K and Grüninger M 2013 Phys. Rev. B 88205115.

[23] De Raychaudhury M, Pavarini E and Andersen O K 2007 Phys. Rev. Lett. 99126402.

[24] Otsuka Y and Imada M 2006 J. Phys. Soc. Jpn. 75124707.

[25] de Groot F 2005 Coord. Chem. Rev. 249 31; Ghiringhelli G, Matsubara M, Dallera C, Fracassi F, Tagliaferri A, Brookes N B, Kotani A and Braicovich L 2006 Phys. Rev. B 73035111.

[26] Laverock J, Chen B, Preston A R H, Smith K E, Wilson N R Balakrishnan G, Glans P-A and Guo J-H 2013 Phys. Rev. B 87125133.

[27] Tanabe Y and Sugano S 1954 J. Phys. Soc. Jpn. 9753.

[28] Khaliullin G, Horsch P and Oleś A M 2001 Phys. Rev. Lett. 86 3879; Oleś A M, Horsch P and Khaliullin G 2007 Phys. Rev. B 75184434.

[29] Pavarini E, Yamasaki A, Nuss J and Andersen O K 2005 New J. Phys. 7188.

[30] Oleś A M, Khaliullin G, Horsch P and Feiner L F 2005 Phys. Rev. B 72214431

[31] Chen B, Laverock J, Newby Jr D, McNulty J, Smith K E, Glans, P-A, Guo J-H, Qiao R M, Yang W, Lees M R, Tung L D, Singh R P and Balakrishnan G 2014 unpublished. 